\title{
REVIEW
}

\section{Acute disseminated encephalomyelitis: recognition in the hands of general paediatricians}

\section{Stonehouse, G Gupte, E Wassmer, W P Whitehouse}

Arch Dis Child 2003;88:122-124

Acute disseminated encephalomyelitis will often present to the general paediatrician as an acute polysymptomatic encephalopathy, and initially the diagnosis may not be clear. A brain MRI scan is essential in establishing the diagnosis and so enabling appropriate advice and treatment to be given. Multicentre clinical audit of outcome and controlled therapeutic trials are needed to secure an evidence base for current practice.

See end of article for authors' affiliations

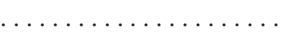

Correspondence to: Dr W P Whitehouse, Senior Lecturer in

Paediatric Neurology,

Academic Division of Child

Health, E Floor East Block,

Queen's Medical Centre,

Nottingham NG7 2UH,

UK; william.whitehouse@

nottingham.ac.uk

Accepted 16 July 2002

Accepted 16 July $2002 \ldots$
A cute disseminated encephalomyelitis (ADEM) is an uncommon, but treatable, inflammatory demyelinating disorder typically affecting the subcortical white matter. Grey matter lesions are seen less often and only in addition to white matter involvement. It is most frequently seen in children and young adults, where it sometimes evolves from an antecedent infection or immunisation. It is usually a monophasic illness, but occasionally it can be multiphasic when the differential diagnosis of multiple sclerosis needs to be considered. It is characterised by a wide range of neurological abnormalities, with the conscious level ranging from normal to coma. Typically the child will present to a general paediatrician with encephalitic signs but non-specific cerebrospinal fluid (CSF) findings. There may be minimal or no changes on the brain computed tomography (CT) scan. Diagnosis is best made using brain magnetic resonance imaging (MRI). This shows the presence of high signal areas of the same age, usually bilateral but asymmetrical, in the hemispheric white matter and elsewhere. These are best seen on T2 weighted or FLAIR images.

Treatment options have included high dose methylprednisolone, ${ }^{1}$ dexamethasone, immunoglobulins, and plasmapheresis. The prognosis is good with most children making a full recovery.

Although ADEM is uncommon it is increasingly being recognised and appears more common than previously thought. The increasing availability of MRI is likely to result in more children being correctly diagnosed and effectively treated.

\section{EPIDEMIOLOGY}

ADEM affects boys and girls equally. ${ }^{2}$ Younger children are more commonly affected. Some studies have shown a seasonal distribution, supporting a link with infectious agents. ${ }^{2}$ The incidence is low, with 3-6 cases presenting a year to a regional neurology centre. ${ }^{23}$ Milder cases are probably managed from time to time, with or without a clear diagnosis, in district general hospitals, and we estimate that a general hospital based paediatrician, with access to acute MRI, will probably see one case a year.

\section{PATHOGENESIS}

ADEM is thought to be an autoimmune disease. ${ }^{4}$ Although infectious agents have been closely associated, no microorganisms have been isolated from the CSF.

Myelin autoantigens, such as myelin basic protein, proteolipid protein, and myelin oligodendrocyte protein could share similar antigenic determinants with those on an infecting pathogen. ${ }^{5}$ The body would then mount an immune reaction, resulting in the production, for example, of antiviral antibodies or a cell mediated response, which cross react with the myelin autoantigens, resulting in the features of ADEM. Studies of children with ADEM have shown that lymphocytes (especially Th2 T cells) have increased reactivity to myelin basic protein. ${ }^{6}$ Furthermore, the pathological similarities of experimental allergic encephalomyelitis (the animal model of inflammatory demyelination) would support the theory that ADEM is of autoimmune aetiology.

\section{PRESENTING FEATURES}

ADEM often follows 7-14 days after a viral infection or immunisation. There may be a prodromal phase with fever, malaise, headache, nausea, and vomiting, which can be followed by meningism and drowsiness. There are multifocal neurological signs including ataxia, tremors, dysarthria, hemiparesis, cranial nerve palsies, optic neuritis, and convulsions, indicating significant involvement of the brain parenchyma, optic nerves, and spinal cord. ${ }^{3}$ Occasionally there may be rapid progression of symptoms and signs to coma and decerebrate rigidity. Rarely ADEM can present as a subtle illness in children with poorly explained irritability, headaches, or an atypical psychiatric illness. ${ }^{78}$ Variations and atypical cases are relatively common, and a rare haemorrhagic variety (Weston-Hurst syndrome) has been described. ${ }^{5}$

\section{PATHOGENS}

Viruses that are associated with ADEM include herpes simplex , HIV, human herpes 6, measles,

\section{Abbreviations: $A D E M$, acute disseminated} encephalomyelitis; CSF, cerebrospinal fluid; CT, computed tomography; MRI, magnetic resonance imaging; MS, multiple sclerosis 
hepatitis A or B, varicella, mumps, influenza, coxsackie, Epstein-Barr, and cytomegalovirus. ${ }^{9}$ Also incriminated in ADEM have been group A $\beta$ haemolytic streptococcus, ${ }^{10}$ Campylobacter, Salmonella, Chlamydia, Mycoplasma, ${ }^{41}$ Legionella, ${ }^{12}$ leptospirosis, Borrelia burgdorferi, and rickettsiae. ${ }^{13} 14$

ADEM has also been reported following immunisation for measles, rubella, meningitis A and $\mathrm{C}^{15}$ influenza, Japanese B encephalitis, smallpox, BCG, and rabies. ${ }^{16}$ Considering the number of immunisations given, ADEM following immunisation is rare. Usually no infectious agent is identified.

\section{INVESTIGATIONS}

There is evidence of inflammation in children with ADEM. Elevation of the white cell count, particularly the lymphocyte count, is common. The erythrocyte sedimentation rate and C reactive protein may also be raised. CSF examination may show a lymphocytosis. CSF protein concentrations are frequently raised, and although most are in the range 0.4-0.6 $\mathrm{g} / \mathrm{l},{ }^{17}$ they can be as high as $2.7 \mathrm{~g} / \mathrm{l}$. Frequently the CSF examination is normal. Oligoclonal bands in the CSF, indicating intrathecal immunoglobulin synthesis, can also be found, but less commonly than in established multiple sclerosis (MS). Serological testing for Epstein-Barr virus, mycoplasma, herpes, varicella, influenza A and B, mumps, cytomegalovirus, and rubella is rarely positive.

The electroencephalogram (EEG) may show an excess of background slow wave activity consistent with the encephalopathy or encephalitic picture. Rarely the EEG may show focal epileptic activity. Visual evoked potentials may show attenuation with degradation and delay in waveform, particularly if there is clinical evidence of bilateral optic neuritis.

\section{NEUROIMAGING}

A cranial CT scan of the brain may be normal, so is often not helpful in establishing a diagnosis. However, MRI of the brain may show early subtle features of the disseminated CNS demyelination associated with $\operatorname{ADEM}^{18}$ (fig 1). MRI T2 weighted, and FLAIR images show the abnormalities more readily than $\mathrm{Tl}$ weighted images. These changes are usually distinguishable from MS. ${ }^{19}$ Involvement of the deep and subcortical white matter is almost universal, whereas grey matter

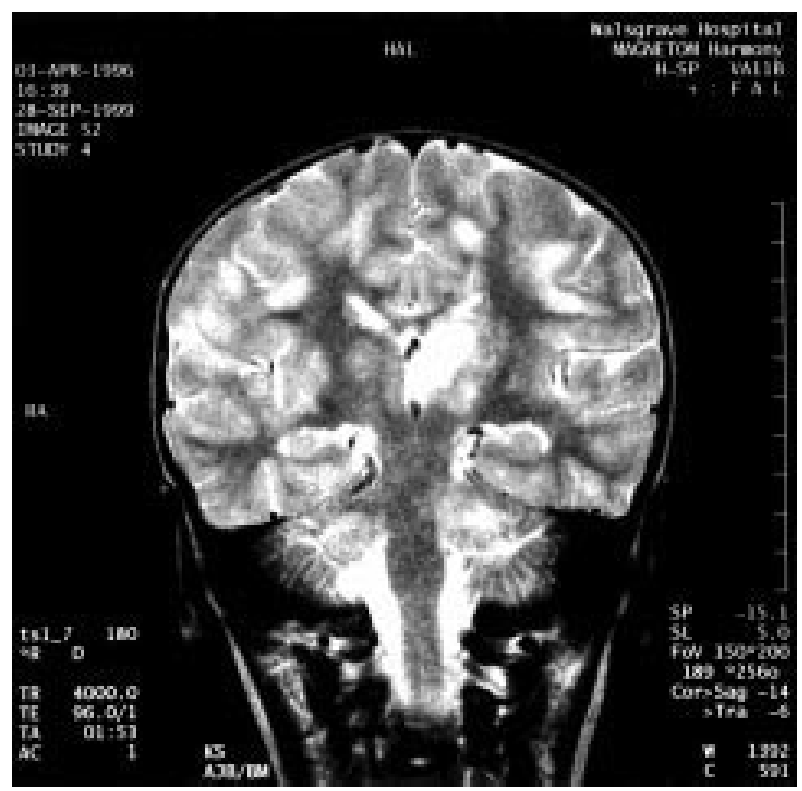

Figure 1 Coronal brain MRI T2 weighted image showing areas of T2 prolongation in subcortical white matter of both cerebral hemispheres, thalamus, cerebellar peduncles bilaterally, dentate nuclei, and upper cervical cord. lesions are seen less often, and only in addition to the more characteristic white matter lesions. Involvement of the thalami and basal ganglia is a typical finding in ADEM, but unusual in MS and may be a useful marker in its differentiation. ${ }^{23}$ In ADEM supratentorial lesions tend to be asymmetrical, whereas thalamic and basal ganglia lesions are often symmetrical. Spinal cord lesions are relatively common too. Additionally the lesions may be extensively distributed.

Follow up MRI scans will show evidence of partial or complete resolution of the lesions and may be useful in differentiating monophasic from multiphasic disease. New lesions or relapses, especially those that occur after six months, may indicate the development of $\mathrm{MS}^{17}$ or relapsing ADEM, which may be considered a special case of MS (see later).

Other techniques not widely available, but helpful in supporting the diagnosis of ADEM, are gadolinium diethylenetriamine penta-acetic acid (Gd-DTPA) enhancement of the lesions on MRI, and areas of increased activity on single photon emission computed tomography. ${ }^{2021}$

\section{TREATMENT}

As most children present with meningism, fever, and an acute encephalopathy, with evidence of inflammation in blood and CSF, they should be covered initially with cefotaxime or other appropriate antibiotic and acyclovir until a diagnosis can be established. Once the diagnosis of ADEM is established, treatment usually commences with 3-5 days of intravenous methylprednisolone (20-30 mg/kg/day), with or without a following course of oral prednisolone commencing at $2 \mathrm{mg} / \mathrm{kg} /$ day and tapering over 4-6 weeks, depending on resolution of clinical signs. In children who have early relapses or in whom there has been a delay in diagnosis, we have used a tapering course of intravenous methylprednisolone over two weeks after the initial course; for example, single dose intravenous methylprednisolone $10 \mathrm{mg} / \mathrm{kg}$, one week after the last dose, followed by another single dose of $5 \mathrm{mg} / \mathrm{kg}$ a week later.

Other treatment options include dexamethasone, intravenous immunoglobulins, ${ }^{22}$ and plasmapheresis, ${ }^{23}$ but little evidence exists for their effectiveness.

\section{ADEM, MULTIPHASIC ADEM, RELAPSING ADEM, AND MULTIPLE SCLEROSIS}

With the absence of a biological marker the distinction between ADEM and MS cannot be made with absolute certainty at the time of first presentation. New lesions or relapses, especially those that occur after six months, may indicate the development of MS. ${ }^{17}$

If the relapse occurs in the months following the initial ADEM, for example during or shortly after steroid withdrawal, the diagnosis of multiphasic acute disseminated encephalomyelitis should be considered. The demyelination can be considered to belong to one multiphasic episode. MS has not yet developed and may or may not develop in the future, with the advent of further episodes of CNS demyelination disseminated in time and space. ${ }^{18}{ }^{23}$

A minority will relapse more than six months later. By operational definition this is a type of MS, fulfilling the criteria of Poser and colleagues ${ }^{24}$ or McDonald and colleagues, ${ }^{25}$ but may be better classified as relapsing acute disseminated encephalomyelitis, a special type of MS. Anecdotal evidence suggests that if relapses are ADEM in characterpolysymptomatic clinically and multifocal on MRI-the prognosis may be better than relapsing remitting MS in general.

In trying to differentiate between ADEM and MS, several pointers may be helpful. ADEM is often associated with a prodromal viral illness. At presentation there may be fever and meningism. These would be unusual in MS. ADEM is typically a monophasic illness producing widespread CNS disturbance, with coma or drowsiness. Conversely MS usually presents as a monosymptomatic syndrome such as optic neuritis or a 
subacute myelopathy, and typically develops a relapsing remitting course. Ataxia is common in ADEM but is rarely a presenting feature in childhood MS.

On reviewing the MRI, ADEM is often associated with a higher lesion load, with larger bilateral but asymmetrical white matter lesions. Plaques in MS are usually smaller and lie in the deep white matter. In ADEM there is thalamic involvement in up to $40 \%$ of cases, while this is rare in MS. The lesions in ADEM are usually of the same age, whereas in MS lesions of different ages (spread in time and space) will be seen. However, complete differentiation between the two diseases is impossible on a single MRI examination. ${ }^{3}$

A few typical ADEM cases will later evolve to typical MS with separate episodes of monosymptomatic demyelination over time. ${ }^{2}{ }^{17}$ Some will relapse with further polysymptomatic ADEM-like demyelination (relapsing acute disseminated encephalomyelitis). In Dale's series, ${ }^{2}$ five of 40 cases presenting with ADEM later had a diagnosis of MS, after a variable follow up with a mean of five years; $10-15 \%$ of cases of ADEM can therefore be expected to relapse and meet diagnostic criteria for MS over about five years. The risk is likely to increase with duration of follow up.

\section{PROGNOSIS}

Most children with ADEM present with an acute aggressive encephalopathy with multifocal neurological deficits. Most make excellent progress over the following days, weeks, or months with no subsequent neurological impairment. Spontaneous remission can occur, although it seems likely that the current practice of active treatment with steroids is usually beneficial. ${ }^{24} \mathrm{~A}$ minority of children are left with a neurological impairment that can range from mild to severe. This can include motor disability, visual impairment, cognitive impairment, behavioural impairment, and epilepsy. Any neurological impairment will need to be addressed, and appropriate coordinated multiagency rehabilitation organised.

\section{Authors' affiliations}

M Stonehouse, E Wassmer, Department of Paediatric Neurology, Birmingham Children's Hospital, Birmingham, UK G Gupte, Department of General Paediatrics, Walsgrave Hospital, Coventry, UK

W P Whitehouse, Academic Division of Child Health, University of Nottingham, Nottingham, UK

\section{REFERENCES}

1 Straub J, Chofflon M, Delavelle J. Early high dose intravenous methyl prednisolone in acute disseminated encephalomyelitis: a successful recovery. Neurology 1997;49:1145-7.
2 Dale RC de Sousa C, Chong WK, et al. Acute disseminated encephalomyelitis, multiphasic disseminated encephalomyelitis and multiple sclerosis in children. Brain 2000;1 23:2407-22.

3 Hynson JL, Kornberg AJ, Coleman LT, et al. Clinical and neuroradiological features of acute disseminated encephalomyelitis in children. Neurology 2001;56:1308-12.

4 Stuve O, Zamvil SS. Pathogenesis, diagnosis, and treatment of acute disseminated encephalomyelitis. Curr Opin Neurol 1999;12:395-401.

5 Fisher RS, Clark AW, Wolinsky JS, et al. Postinfectious leukoencephalitis complicating Mycoplasma pneumoniae infection. Arch Neurol 1983:40:109-13

6 Phole-Koppe A, Burchett SK, Thiele EA, et al. Myelin basic protein reactive Th2 T cells are found in acute disseminated encephalomyelitis. J Neuroimmunol 1998;91:19-27.

7 Nasr JT, Andriola MR, Coyle PK. ADEM: literature review and case report of acute psychosis presentation. Pediatr Neurol 2000;22:8-18

8 Patel SP, Friedman RS. Neuropsychiatric features of acute disseminated encephalomyelitis: a review. J Neuropsychiatry Clin Neurosci 1997:9:534-40

9 Kanzaki A,Yabuki S. Acute disseminated encephalomyelitis associated with cytomegalovirus infection - a case report. Rinsho Shinkeigaku 1994;3:51 1-13

10 Hall MC, Barton LL, Johnson MI. Acute disseminated encephalomyelitis like syndrome following group $A \beta$ hemolytic streptococcal infection. J Child Neurol 1998;13:354-6.

11 Yamamoto K, Takayanagi M, Yoshihara, et al. Acute disseminated encephalomyelitis associated with Mycoplasma pneumonia infection. Acta Paediatr Japon 1996;38:46-51.

12 Easterbrook PJ, Smyth EG. Postinfectious encephalomyelitis associated with Mycoplasma pneumonia and Legionella pneumophila infection. Postgrad Med J 1992;68:124-8.

13 Wei TY, Baumann RJ. Acute disseminated encephalomyelitis after Rocky Mountain Spotted fever. Pediatr Neurol 1999;21:503-5.

14 Hartung HP, Grossman RI. ADEM-distinct disease or part of the MS spectrum? Neurology 2001;56:1257-60.

15 Py MO, Andre C. Acute disseminated encephalomyelitis and meningococcal $A$ and $C$ vaccine: a case report. Arq Neuropsiquitr 1997;55:632-5.

16 Apak AR, Kose G, Anlar B, et al. Acute disseminated encephalomyelitis in childhood: report of 10 cases. J Child Neurol 1999;14:198-201.

17 Schwartz S, Mohr A, Knauth M, et al. Acute disseminated encephalomyelitis. A follow-up study of 40 adult patients. Neurology $2001 ; 56: 1313-18$

18 Sztajnbok J, Lignani L, Bresolin AU, et al. Acute disseminated encephalomyelitis: an unusual cause of encephalitic syndrome in childhood. Pediatr Emerg Care 1998;14:36-8.

19 Kesselring J, Miller DH, Robb SA, et al. Acute disseminated encephalomyelitis MRI findings and the distinction from multiple sclerosis. Brain 1990;113:291-302

20 Caldermeyer KS, Harris TM, Smith RR, et al. Gadolinium enhancement in acute disseminated encephalomyelitis. J Comput Assist Tomogr $1991 ; 15: 673-5$

21 Broitch $\mathbf{K}$, Horwich D, Alavi A. HMPAO-SPECT and MRI in acute disseminated encephalomyelitis. J Nucl Med 1991;32:1897-900.

22 Sahlas DJ, Miller SP, Guerin M, et al. Treatment of acute disseminated encephalomyelitis with intravenous immunoglobulin. Neurology 2000;54:1370-2.

23 Kanter DS, Horensky D, Sperling RA, et al. Plasmapheresis in fulminant acute disseminated encephalomyelitis. Neurology 1995;45:824-7.

24 Poser CM, Paty DW, Scheinberg L, et al. New diagnostic criteria for multiple sclerosis: guidelines for research protocols. Ann Neurol 1983; 13:227-31.

25 McDonald WI, Compston A, Edan G, et al. Recommended diagnostic criteria for multiple sclerosis: guidelines from the international panel on the diagnosis of multiple sclerosis. Ann Neurol 2001;50:121-7. 